\title{
FIRMST2020-AB08 \\ OXYTOCIN PATHWAY POLYMORPHISMS AND SOCIAL FUNCTIONING IN SCHIZOPHRENIA
}

Peer reviewed by JS Bamrah, Ananthakrishnan Raghuraman, Soumit Dasgupta \& Jyothi Srinivasan

\section{Aim:}

To find the risk alleles for poor social functioning in the oxytocin pathway polymorphisms in patients with schizophrenia.

Keywords

Oxytocin; schizophrenia; social cognition; stress factors

\begin{abstract}
Vera Mikhailova
I.M. Sechenov First Moscow State Medical University, Moscow, Russian Federation

Correspondence to voviand.vm@gmail.com

Cite as: Mikhailova, V. (2020) OXYTOCIN PATHWAY POLYMORPHISMS AND SOCIAL FUNCTIONING IN SCHIZOPHRENIA. The Physician vol 6; issue 3; epub 21 Aug 2020; DOI: https://doi.org/10.38192/1.6.3.firmst20.ab08

Article Information

Published 21 Aug 2020

Open Access - Creative Commons Licence CC-BYND-4.0
\end{abstract}

\section{Introduction}

The social adaptation for any schizophrenia patient and their family is the main factor allowing to judge whether therapy is working or not. That makes the improvement of patients' social functioning the main goal for an attending physician, the pharmaceutical industry specialists and also for biomedical researchers.

To obtain a better knowledge of the pathogenesis of poor social performance in schizophrenia patients under treatment, it seems necessary to take into consideration the disturbance of the oxytocin pathway.

Neurohormone oxytocin is known to play an important role in social behaviour. The latest neuroimaging studies have proven OXTR (oxytocin receptor) expressing cells to be largely involved in social cognition tasks. Moreover, such polymorphisms as CD38 (rs3796863), OXTR ( rs53576), OXTR ( $r$ 4686302) have been observed to be associated with the pathogenesis of several diseases such as autism, OCD and bipolar disorder.

\section{Methods}

Social functioning was assessed with the Personal and the Social Performance (PSP) scale in 732 patients (women 343), aged $36.5 \pm 12.3$ years, with an ICD-10 diagnosis of schizophrenia or schizoaffective psychosis. Firstly, genotyping was performed with HRM (High Resolution Melt) method using DNA samples extracted from patients' venous blood samples by organic solvents and amplificated by PCR.

Secondly, all patients were stratified by the level of social functioning into two groups: "moderate and good" and "marked and poor". Allele frequencies were aligned with the Hardy-Weinberg equation ( $p>0,05)$. Genotypes were calculated in each group of social functioning.

\section{Results}

CC allele CD38 (rs3796863) tended to be more frequent in "poor" performance group within a socially useful activity PSP scale. OXTR rs53576 GG allele was considerably more frequent in "poor" performance 
group within the interpersonal relations PSP scale.

\section{Conclusion}

It is, therefore, the first-time oxytocin pathway polymorphisms are found to be associated with social functioning in schizophrenia patients, with CD38 rs3796863 CC and OXTR rs53576 GG being risk allele.

Conflict of Interest

No conflict of interest declared 